\title{
Clinical effectiveness of baclofen for the treatment of alcohol dependence: a review
}

This article was published in the following Dove Press journal:

Clinical Pharmacology:Advances and Applications

2 July 2013

Number of times this article has been viewed

\author{
Jessica L Brennan² \\ Jonathan G Leung' \\ Jane P Gagliardi ${ }^{3}$ \\ Sarah K Rivelli ${ }^{3}$ \\ Andrew J Muzyk ${ }^{4}$ \\ 'Department of Hospital Pharmacy \\ Services, Mayo Clinic, Rochester, MN, \\ ${ }^{2}$ Western Psychiatric Institute and \\ Clinic, University of Pittsburgh Medical \\ Center, Pittsburgh, PA, ${ }^{3}$ Department \\ of Psychiatry and Behavioral Sciences, \\ Duke University, Durham, NC, \\ ${ }^{4}$ Department of Pharmacy Practice, \\ Campbell University School of \\ Pharmacy and Health Sciences, Buies \\ Creek, NC, USA
}

Correspondence: Andrew J Muzyk Department of Pharmacy Practice, Campbell University School of Pharmacy and Health Sciences, PO Box 3089 Pharmacy, Durham 277I0, NC, USA Email andrew.muzyk@duke.edu

\begin{abstract}
Baclofen, an agonist at the B subunit of gaba-aminobutyric acid receptor, possesses pharmacologic properties that may confer utility for the treatment of alcohol dependence. Research suggests that not only can it be useful in promoting maintenance of alcohol abstinence but also it may play a key role in decreasing alcohol cravings and anxiety often associated with alcohol dependence. To assess the benefit of baclofen for alcohol dependence, a review of the literature was conducted to identify published data investigating this off-label treatment. Four randomized controlled trials to date have been published and were included in this review. Although primary outcomes differ between studies, patients randomized to baclofen experience higher rates of abstinence from alcohol than those taking placebo in two of the trials. Secondary analyses indicate that baclofen is safe in patients with alcohol dependence, including those with moderate to severe liver cirrhosis, and may provide beneficial anxiolytic effects. Despite some positive data, the largest available randomized controlled trial failed to find any differences between baclofen and placebo. In all studies, individuals with severe medical comorbidities, seizure disorders, and psychiatric disorders were excluded from trials, which may limit external validity. In summary, there may be beneficial effects from using baclofen for the treatment of alcohol dependence; however, limited conclusions can be drawn from the small number of studies currently available for review. Larger well-designed trials are needed to further define baclofen's role for the treatment of alcohol dependence.
\end{abstract}

Keywords: baclofen, alcohol, abstinence, relapse, craving, dependence

\section{Introduction}

Alcohol misuse is one of the world's leading health risks. Alcohol abuse and dependence are associated with more than 60 disease types and injuries that contribute to approximately 2.5 million deaths annually. ${ }^{1}$ An estimated $4.5 \%$ of the global burden of disease and injury is attributed to alcohol. Alcohol is also associated with exorbitant social costs, such as those related to violence, child neglect/abuse, and absenteeism in the workplace. ${ }^{1}$ Therapeutic approaches, including pharmacotherapy, play a pivotal role in treating patients with alcohol use disorders but are commonly underutilized. ${ }^{2}$ Since the 1951 approval of disulfiram by the Food and Drug Administration (FDA), three additional treatments have been approved for the treatment of alcohol dependence: acamprosate, oral naltrexone, and intramuscular naltrexone. ${ }^{3}$

Despite mixed data from well-designed trials of disulfiram, acamprosate, or naltrexone, two recent Cochrane reviews concluded that acamprosate and naltrexone appear to be safe and effective in patients with alcohol dependence, with moderate effect sizes. ${ }^{4-8}$ However, due to various barriers to the use of acamprosate, 
naltrexone, or disulfiram, some patients are unable to take these FDA-approved agents. Acamprosate is contraindicated in patients with severe renal impairment (ie, estimated creatinine clearance $[\mathrm{CrCl}]<30 \mathrm{~mL} / \mathrm{min}$ ), and gastrointestinal adverse events may prevent use or limit dose maximization. Naltrexone is contraindicated in patients with acute hepatitis or hepatic failure and may not be used in patients with concomitant opioid utilization. Disulfiram must be used with caution in multiple disease states, such as diabetes mellitus, hypothyroidism, and seizures; patients must also be warned not to consume "hidden" alcohol, such as tonics and mouthwashes, in order to avoid unintended toxic effects of the medication. Nonadherence with oral naltrexone remains a barrier to effective treatment for a large portion of patients, so intramuscular naltrexone has been developed for oncemonthly administration and has additional advantages of steady therapeutic drug concentration and decreased peak and trough effects. ${ }^{9}$

The off-label use of multiple medications, including baclofen, has been investigated for maintenance of abstinence from alcohol. Baclofen was originally approved by the FDA in 1977 for use in spasticity associated with neurologic conditions, such as multiple sclerosis and spinal cord lesions. ${ }^{10}$ Baclofen's pharmacologic properties have led to investigation of its benefit for the treatment of alcohol dependence.

\section{Baclofen: pharmacokinetics and pharmacodynamics}

Baclofen is an agonist at the $\mathrm{B}$ subunit of gaba-aminobutyric acid receptor $\left(\mathrm{GABA}_{\mathrm{B}}\right)$. $\mathrm{GABA}_{\mathrm{B}}$ receptors are metabotropic and differ from the ionotropic $\mathrm{GABA}_{\mathrm{A}}$ and $\mathrm{GABA}_{\mathrm{C}}$ receptors. $\mathrm{GABA}_{\mathrm{B}}$ receptors are highly expressed in the limbic system, suggesting a major role in regulating emotional behavior. GABA neurotransmission is also known to be an important factor in the control of anxiety. Following these lines of evidence, activation of $\mathrm{GABA}_{\mathrm{B}}$ receptors might reduce anxiety. ${ }^{11}$ Noting that anxiety is a common symptom in patients being treated for alcohol withdrawal and alcohol dependence, activating $\mathrm{GABA}_{\mathrm{B}}$ receptors might be useful in the treatment of alcohol withdrawal and dependence.

Mesolimbic dopamine neurons, found in the same areas of the brain as $\mathrm{GABA}_{\mathrm{B}}$ receptors, are thought to be involved in the mediation of alcohol intake and reinforcement. Activation of $\mathrm{GABA}_{\mathrm{B}}$ receptors by baclofen may result in local inhibition of surrounding dopamine neurons. Through this mechanism, alcohol-stimulated dopamine release would be reduced, thereby decreasing positive reinforcement from alcohol consumption and aiding in abstinence from alcohol. ${ }^{12}$
Despite high individual variation in pharmacokinetics, baclofen is rapidly and extensively absorbed from the gastrointestinal tract following oral administration, though there is some suggestion that absorption is inversely related to dose. ${ }^{13,14}$ Peak plasma concentrations are generally observed 2-3 hours after ingestion. Baclofen's elimination half-life is 2-6 hours and it is usually dosed three to four times daily. ${ }^{13}$ Baclofen is excreted primarily unchanged by the kidneys, making it a useful agent in patients with impaired hepatic function or a high potential for hepatic cytochrome P450mediated drug-drug interactions.

\section{Evidence for baclofen in maintenance of alcohol dependence: a critical evaluation}

Numerous case reports, case series, and open-label trials have been published evaluating baclofen for the treatment of alcohol dependence; most of the primary outcomes focus on abstinence from alcohol (Table 1.) Limited data are available from well-conducted trials, though numerous trials are underway or recently completed with a goal of evaluating baclofen for maintenance of abstinence in alcohol-dependent patients. ${ }^{15}$ A search of MedLine (1966-2013) using the terms "baclofen," "alcohol," "abstinence," "relapse," "craving," and "dependence" was conducted to identify English language articles. Randomized controlled trials (RCTs) are evaluated in the following text and summarized in Table 2.

Addolorato et $\mathrm{al}^{16}$ conducted an RCT evaluating the effectiveness and safety of baclofen for maintenance of alcohol abstinence specifically in patients with cirrhosis of the liver. All subjects had a biopsy-proven diagnosis of cirrhosis; their alcohol intake included at least two heavy drinking days per week, with an average of at least 21 drinks weekly for men and 14 drinks weekly for women. Exclusion criteria included the presence of severe cardiac or pulmonary disease. Individuals with other addictions (except nicotine), pharmacologically treated psychiatric diagnoses, epilepsy, a severe medical illness, renal dysfunction, or the use of interferon or corticosteroids within 60 days of enrolment were also excluded. Subjects were initially hospitalized for 3-4 days to complete a medical evaluation and treatment for alcohol withdrawal syndrome (AWS) with diazepam. After resolution of AWS, subjects were eligible for randomization. Eightyfour subjects with a median age of 56 years (interquartile range 45.5-62 years) were included in this study. The active treatment group received baclofen $10 \mathrm{mg}$ orally three times daily. Assessments and counseling sessions occurred weekly for 4 weeks and then biweekly for the remainder of the 


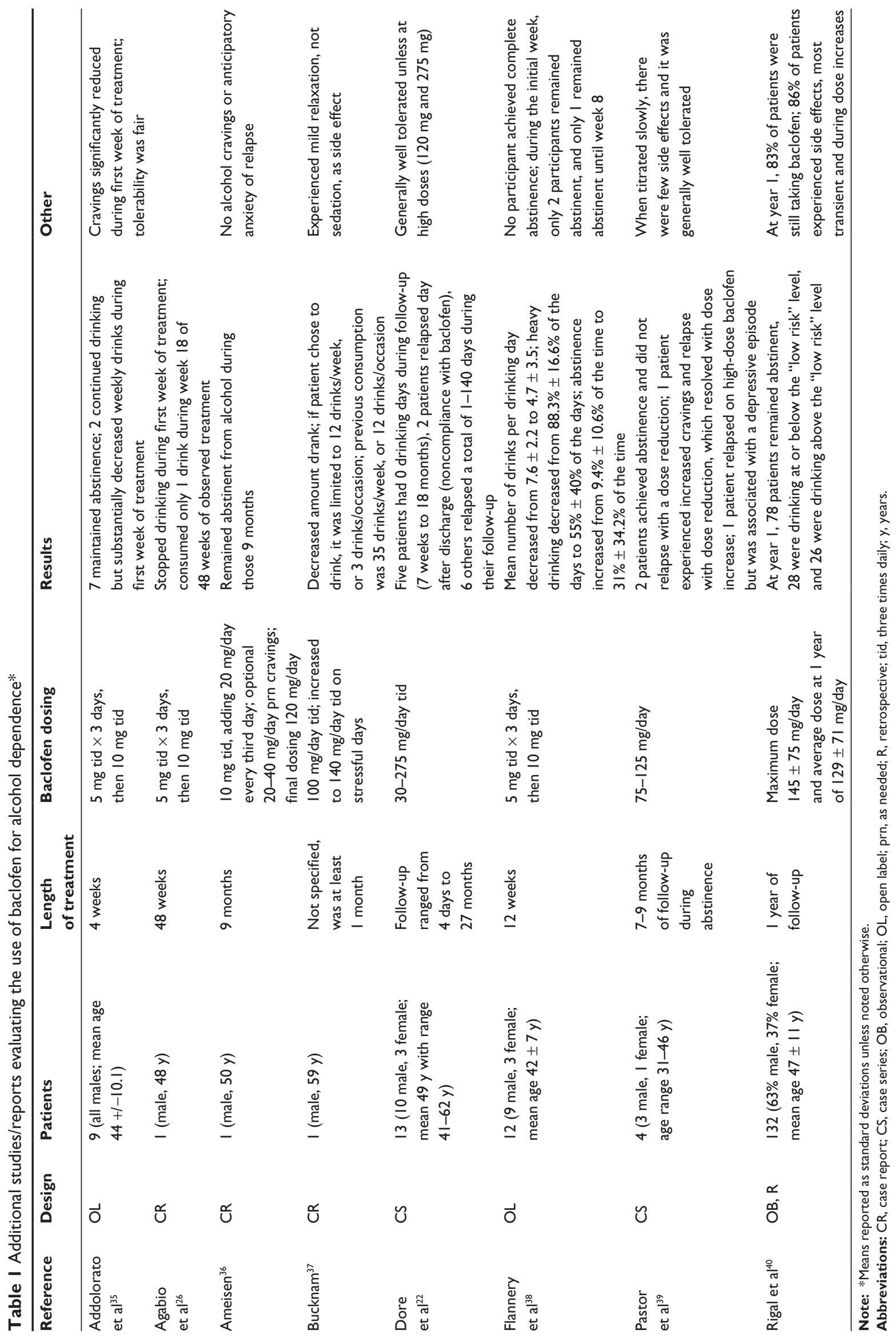


Table 2 Description of randomized controlled trials

\begin{tabular}{|c|c|c|c|}
\hline Source & Addolorato et al ${ }^{16}$ & Garbutt et al ${ }^{18}$ & Addolorato et al ${ }^{19}$ \\
\hline Setting & Single inpatient site & Outpatients recruited from community & Single inpatient site \\
\hline \multicolumn{4}{|l|}{ Subjects } \\
\hline Number & 84 & 80 & 39 \\
\hline Age $(y)$ & 49.0 (range 43.0-6I.0) & $47.5 \pm 7.6$ & $45.8 \pm 10.6$ \\
\hline Male gender & $76 \%$ & $55 \%$ & Not reported \\
\hline Daily drinks & Not reported & $7.3 \pm 3.7$ (baclofen group) & I $4.2 \pm 7.9$ (all subjects) \\
\hline Duration of use $(y)$ & 22.0 (range 17.0-27.0) & $23.5 \pm 9.9$ & $12.6 \pm 4.8$ \\
\hline Comorbidities & Cirrhosis (Child-Pugh A, B, or C) & No medical/psychiatric & No medical/psychiatric \\
\hline Intervention & Baclofen $10 \mathrm{mg}$ po tid for 12 weeks & Baclofen $10 \mathrm{mg}$ po tid for 12 weeks & Baclofen $10 \mathrm{mg}$ po tid for 4 weeks \\
\hline \multirow[t]{3}{*}{$\begin{array}{l}\text { Primary outcome/ } \\
\text { results }\end{array}$} & $\begin{array}{l}\text { Percentage of patients maintaining } \\
\text { abstinence: } 71 \% \text { vs } 29 \%(P=0.000 \mathrm{I})\end{array}$ & $\begin{array}{l}\text { Percentage of heavy drinking days: } \\
19.3 \% \text { vs } 24.7 \%(P=0.73)\end{array}$ & $\begin{array}{l}\text { Percentage of patients maintaining } \\
\text { abstinence: } 70 \% \text { vs } 21.2 \%(P<0.005)\end{array}$ \\
\hline & Cumulative abstinent days: & Percentage of abstinent days: & Cumulative abstinent days: \\
\hline & $62.8 \pm 5.4$ vs $30.8 \pm 5.5(P=0.00 I)$ & $51.7 \%$ vs $51.6 \%(P=0.6 I)$ & $19.6 \pm 2.6$ vs $6.3 \pm 2.4(P<0.005)$ \\
\hline
\end{tabular}

Note: Reported as mean + standard deviation unless noted otherwise.

Abbreviations: po, orally; tid, three times daily; vs, versus; $y$, years.

12-week study period. The primary outcome was the effect of baclofen on total abstinence and cumulative abstinence duration. Craving level was assessed using a version of the Obsessive Compulsive Drinking Scale.

A greater proportion of subjects maintained abstinence in the baclofen group compared with the placebo group ( $71 \%$ versus [vs] 29\%; odds ratio [OR] 6.3 [95\% confidence inter$\operatorname{val}(\mathrm{CI}) 2.4-16.1])$. There was also a greater mean cumulative days abstinent in the treatment group (62.8 days) versus the placebo group (30.8 days) $(P=0.001)$. Survival analysis demonstrated a lower rate of 60-day relapse with baclofen treatment as compared with placebo (hazard ratio 0.4 [95\% CI 0.2-0.8]). Subjects with Child-Pugh class B and C cirrhosis assigned to the baclofen group were more likely to abstain from alcohol altogether than those in the placebo group (OR 4.5 [95\% CI 1.2-17.4] and 8.3 [95\% CI 1.7-41.3]), respectively. No significant difference was observed for subjects with Child-Pugh class A cirrhosis. Baclofen treatment did result in a statistically significant improvement in Obsessive Compulsive Drinking Scale scores, suggesting less craving for alcohol in those on baclofen therapy. Laboratory values improved among those randomized to baclofen and were consistent with decreased alcohol use, reflected by decreased transaminases and improved markers of synthetic liver function. Baclofen was well tolerated and there were no life-threatening adverse events reported with baclofen use (headache, $n=4$; vertigo, $n=2$; tiredness, $n=1$; sleepiness, $\mathrm{n}=1$ ) or with its discontinuation at the end of the treatment phase.

A post hoc analysis from the same study evaluated only subjects with a secondary diagnosis of hepatitis $\mathrm{C}$ virus (HCV) infection. ${ }^{17}$ As with the entire cohort, subjects with HCV demonstrated a greater likelihood of total alcohol abstinence when taking baclofen $(83.3 \%)$ versus placebo (25\%); $P=0.01$. However, these results should be interpreted with caution, given the limitations of post hoc analyses, with additional well-conducted studies needed to confirm the safety and efficacy in patients with HCV infection.

Based on the data from Addolorato et al, ${ }^{16}$ baclofen appears to be a well-tolerated and effective medication for the promotion of alcohol abstinence in patients with cirrhosis. It is important to note limitations of the study, however, including limited ability to generalize the findings to other patient populations (limited external validity), given the narrow inclusion and exclusion criteria. In addition to problems with external validity, the study exhibits challenges related to statistical inference and missing data. For instance, although various laboratory parameters were reported as statistically different from group to group, it is difficult to ascertain clinical significance without information about the actual laboratory values in the two groups. Additionally, it is unclear whether bias was introduced due to the use or choice of benzodiazepine used to treat initial symptoms associated with AWS. Although a similar number of patients received treatment with diazepam (13 subjects in the baclofen group received diazepam vs eleven in the placebo group), the mean dosage received was not available. Given that diazepam has a long elimination half-life, which may be additionally prolonged in liver disease, its use may have impacted craving for alcohol and symptoms of anxiety and irritability traditionally associated with withdrawal.

Garbutt et a $1^{18}$ conducted a double-blind RCT assessing the efficacy and safety of a total daily dose of $30 \mathrm{mg}$ of baclofen for alcohol dependence. The primary outcomes of the study were number of heavy drinking days and proportion of abstinence between groups. Other outcomes included 
percentage abstinent days, time to usage, craving, anxiety, depression, and safety information. Individuals with a diagnosis of alcohol dependence and at least two heavy drinking days weekly during the 4 weeks prior to enrolment were eligible for participation. Those with significant medical comorbidities, including seizure disorder, psychiatric diagnoses, or abnormal hepatic or renal laboratory values, were excluded. Concomitant use of insulin, oral antihyperglycemics, anticonvulsants, and psychotropics (except stable doses of antidepressants) was not permitted. Subjects were evaluated at baseline and weekly for 4 weeks and then twice monthly for the remainder of the 12-week study period. They also received eight psychosocial sessions during the study time frame. Eighty subjects were included; randomization was stratified by sex. Of the 44 men, 22 received baclofen and 22 received placebo. A total of 36 women were included, and 18 were randomized to baclofen therapy.

In the baclofen group, $25.5 \% \pm 23.6 \%$ of days over the 12-week period were described as heavy drinking days; this was statistically indistinguishable from the $25.9 \% \pm 23.6 \%$ of days described as heavy drinking days in the placebo group $(P=0.56)$. There were no differences between groups in terms of weekly heavy drinking days, percentage abstinent days, or for the secondary outcomes of craving, depression, or anxiety. Though the study failed to demonstrate benefit from the drug, baclofen was well tolerated with no significant reported adverse events. Drowsiness (placebo, $n=4$; baclofen, $n=11$ ) and headache (placebo, $n=4$; baclofen, $\mathrm{n}=1$ ) were the most commonly reported events and did not differ between groups. A limitation in this study relates to the stringent exclusion criteria, which may have excluded subjects who could have derived benefit and also limits generalizability (ie, a threat to external validity). Moreover, subjects in this study may have been less severely dependent on alcohol than in the Addolorato study, and baclofen may have more impact on more dependent individuals.

The two aforementioned trials were conducted in response to open-label data, and a preliminary 30-day double-blind RCT conducted by Addolorato et $\mathrm{al}^{19}$ in 2002 at the same facility from which the 2007 data were obtained. In this trial, 39 subjects with a diagnosis of alcohol dependence were randomized to receive placebo or baclofen titrated to a total daily dose of $30 \mathrm{mg}$ by day 4 of the study. To be included in the study, subjects were required to have consumed alcohol within 24 hours of enrolment, and they were required to have a family member available to participate in follow-up visits and to ensure adherence. Individuals with severe kidney, liver, or heart disease were excluded, as were those with psychopathologic illnesses, addiction to other substances (except nicotine), and seizure disorders or those using antiepileptic medications. After randomization, subjects could receive emergent diazepam for symptoms associated with AWS, though none required this intervention. Subjects were evaluated weekly for outcomes related to abstinence using patient self-report and alcohol concentration. Cravings, biologic markers of alcohol abuse, anxiety, depression, and adverse events during the trial were also assessed on a weekly basis.

A greater percentage of subjects in the baclofen group (70\%) achieved and maintained abstinence versus the placebo group $(21.1 \%, P<0.005)$. The cumulative days abstinent ( \pm standard error of the mean) over the 4-week period was also greater in the baclofen group, $19.6 \pm 2.6$ days and $6.3 \pm 2.4$ days $(P<0.005)$. Mean number of daily drinks decreased over the course of 2 weeks from 18 to less than 0.5 in the baclofen group, compared with a decrease from ten to four in the placebo group. Secondary outcomes of cravings and anxiety were significantly reduced in those treated with baclofen as compared with placebo, but scores for depressive symptoms did not differ between the two groups. No statistical analysis was conducted on biologic markers of hepatic function; however, both groups had reductions of gammaglutamyl transpeptidase, aspartate aminotransferase, and alanine transaminase by approximately $50 \%$ from baseline. Baclofen was well tolerated, with no discontinuations for adverse events. The most commonly reported side effect in the treatment group was sleepiness $(n=2)$. The duration of the trial was short, leading to the evaluation of baclofen in longer trials with larger sample sizes.

Information regarding a fourth double-blind RCT, which planned to evaluate the effect of baclofen on the maintenance of abstinence, has been described. ${ }^{10}$ However, data are available only from a post hoc analysis in a separate publication, due to protocol violations and recruitment issues. ${ }^{20}$ The International Baclofen Intervention Study (IBIS) attempted to recruit subjects from four European countries. Baclofen $60 \mathrm{mg}$ daily, baclofen $30 \mathrm{mg}$ daily, and placebo groups were compared for the primary outcomes of heavy drinking days, abstinence days, and alcohol craving. Assessments were made at weekly intervals for the first 4 weeks and then decreased to every other week for the remainder of the 12-week trial. There was also a final follow-up visit at week 16, 4 weeks after the last dose of baclofen or placebo. At enrolment, patients were assessed and treated for AWS with diazepam when necessary. Those requiring AWS treatment for greater than 10 days were excluded. Brief psychological 
intervention was provided and medication adherence was assessed at each follow-up assessment.

Due to protocol violations and missing data, authors excluded 51 subjects from Australian and Austrian study sites, and a third site in the UK did not enrol any patients. Primary analysis of the IBIS study data is commented upon in a second publication ${ }^{20}$ and reported that no significant differences were found for primary outcomes but noted that due to exclusions the study failed to meet power.

In this second publication is also a post hoc analysis of the 42 Italian subjects meeting the inclusion and exclusion criteria of the IBIS study. Reported as incidence rate ratios when compared with placebo, baclofen $30 \mathrm{mg}$ was reported to have a $53 \%$ reduction of daily drinks and baclofen $60 \mathrm{mg}$ daily a $68 \%$ reduction of daily drinks $(P<0.001)$. The authors concluded that there was an association between increasing dose and increasing effect $(P=0.0214)$. This study utilized doses higher than those previously studied for this indication; however, all treatment groups continued to have minimal adverse events, with headache and sleepiness those most commonly reported. Due to the small number of subjects and the post hoc nature, it is difficult to draw firm conclusions regarding the benefit of $60 \mathrm{mg}$ compared with $30 \mathrm{mg}$ of baclofen for this indication. Further well-conducted studies are needed to confirm whether higher doses of baclofen may confirm greater efficacy.

\section{Is baclofen safe for maintenance of alcohol dependence?}

Baclofen has been used safely for decades as an antispasticity medication. Only recently has the understanding of the pathophysiology of alcohol dependence evolved, leading to the evaluation of baclofen's role for the maintenance of alcohol dependence. Although data are mixed regarding baclofen's efficacy in alcohol dependence, data are consistent in terms of safety. The most commonly reported adverse events include headache, drowsiness, nausea, and vertigo. ${ }^{11,18-20}$ In addition to central nervous system effects, nausea may occur in up to $12 \%$ of patients but can be minimized by gradual titration to a target dose or taking baclofen with food. ${ }^{21}$ No difference in adverse events between the $30 \mathrm{mg}$ and $60 \mathrm{mg}$ daily dosing has been identified. ${ }^{20}$ Literature supporting higher doses of baclofen, mostly case reports and series, has reported the safe use of doses up to $275 \mathrm{mg}$ daily. ${ }^{22}$ However, there are very limited safety data utilizing highdose baclofen in alcohol-dependent patients, and significant adverse events have been reported, including overdose and seizures. ${ }^{21-23}$
Evidence also supports baclofen as a safe maintenance treatment for alcohol dependence, even when patients continue to consume alcohol or resume drinking alcohol. Evans and Bisaga ${ }^{24}$ studied 18 heavy social drinkers who consumed intoxicating levels of alcohol and demonstrated no adverse effects from the combination of alcohol and baclofen. Rare but serious adverse effects associated with baclofen in other uses, including encephalopathy, seizure, and hyperammonemia, have not been identified in clinical trials investigating its use for alcohol dependence. ${ }^{11,18-20}$ No negative sequelae have been reported with baclofen discontinuation in alcohol dependence studies, though seizures and other neuropsychiatric symptoms have been reported after abrupt discontinuation of oral baclofen when used for other indications. ${ }^{21}$ The lack of noted adverse events may be a result of small sample sizes and low doses of baclofen utilized in available trials, and clinicians should remain mindful of possible rare adverse events associated with baclofen. However, with available data suggesting safety, further investigation of baclofen's role in the treatment of alcohol dependence is warranted.

\section{Discussion}

The evidence supporting the safe and effective use of baclofen for maintenance treatment of alcohol dependence is growing, as evidenced by the number of ongoing or recently completed trials. From the studies reviewed here, it appears that baclofen may prolong the time to first drink, reduce overall drinking days, and facilitate maintenance of abstinence in select populations. Baclofen appears to be well tolerated at the doses that have been evaluated. The available evidence suggests a potentially unique impact of baclofen for the treatment of alcohol dependence, given that it has been safely used in patients with comorbid cirrhosis of the liver, a disease that is not uncommon in alcohol-dependent patients. However, the role of baclofen in patients with other medical and psychiatric comorbidities remains unclear. With the exception of stable antidepressant use, those with a treated comorbid psychiatric illness were excluded from RCTs. As the efficacy of baclofen has not been conclusively confirmed for the maintenance of alcohol abstinence, excluding subjects taking other psychotropic medications may avoid confounding from other psychotropic agents that may have beneficial effects on alcohol intake. However, baclofen may provide clinical benefit in those with underlying anxiety, and expanding future inclusion criteria in clinical trials would be important to test this hypothesis. Addolorato et al ${ }^{19}$ found improvements in anxiety but not depression scores in patients treated with baclofen. The anxiolytic effects of 
baclofen have also been shown to be greater than placebo in alcoholic patients, producing similar results to both low-dose amitriptyline and diazepam, though in that particular study abstinence from alcohol was not measured or reported. ${ }^{25}$ One case report describes a patient with schizophrenia and alcohol dependence who experienced decreased alcohol intake and cravings when he was treated with baclofen. ${ }^{26}$ Additionally, Dore et $\mathrm{al}^{22}$ reported a case series of baclofen use in dually diagnosed individuals $(n=13)$. When doses ranging from $30 \mathrm{mg}$ to $275 \mathrm{mg}$ of baclofen were used daily, eight (61.5\%) subjects experienced a significant abstinence period or reduction in drinking quantity. Four subjects also reported beneficial anxiolytic effects. Unfortunately, two subjects overdosed with baclofen, and seven patients experienced mild to moderate adverse events, including drowsiness, nocturnal enuresis, forgetfulness, and dizziness. Additional data supporting the efficacy and safety of baclofen in dually diagnosed patients are warranted and necessary before it can be firmly recommended over other potential options.

RCTs of baclofen for the maintenance of alcohol abstinence also excluded patients with documented seizure disorders. However, it is unclear whether individuals with alcohol-related seizures were also excluded. It has been estimated that at least $15 \%$ of patients with alcohol dependence have a documented seizure history. Alcohol itself is a significant risk factor for the development of status epilepticus. ${ }^{27}$ Though prospective trials of baclofen for alcohol dependence have not demonstrated increased seizure risk, the package insert and other trials report a risk of baclofen-related seizures at doses greater than $200 \mathrm{mg}$ per day. ${ }^{13,28}$ Albeit a rare event, the association of seizures with baclofen withdrawal and overdose should bring awareness for the potential for seizure-related complications. This fact was highlighted in one case report describing a patient with no seizure history who developed two seizures while being treated with baclofen $240 \mathrm{mg}$ daily for the maintenance of alcohol abstinence. ${ }^{23}$

An additional population excluded in the trials discussed was those with renal dysfunction. Renal impairment can lead to the accumulation of baclofen, resulting in increased adverse events. Currently, there are no manufacturer recommendations for renal dose adjustments for baclofen. There are also limited data on baclofen toxicity in patients with varying degrees of renal impairment. El-Husseini et al ${ }^{14}$ proposed that baclofen not be given to those with $\mathrm{CrCl}<30 \mathrm{~mL} / \mathrm{min}$ and be initiated and titrated slowly when $\mathrm{CrCl}$ is $30-50 \mathrm{~mL} / \mathrm{min}$. It has also been suggested to use caution in patients with subclinical or mild renal impairment, as serum concentration of stable baclofen doses has been observed to increase over time. $^{29}$

Given the limited external validity and methodological limitations of current literature, clinicians should be aware of other medications studied off label for maintenance of alcohol abstinence. Although an extensive review of such agents is beyond the scope of this article, medications that have been studied include antiepileptic agents. Topiramate, gabapentin, and pregabalin have also been studied in RCTs and have demonstrated benefits for decreasing the percentage of heavy drinking days, percentage of days abstinent from alcohol, and number of drinks per day. 2,30,31 These medications have the advantages of increasing the seizure threshold and having specific FDA-approved renal dosing recommendations for patients with renal impairment. Gabapentin and pregabalin have also been shown to have beneficial effects on anxiety. Limitations to the use of these agents include adverse cognitive effects that may limit topiramate titration to an optimal dose, and the rare occurrence of euphoria associated with pregabalin.

Other medications, such as quetiapine, a second-generation antipsychotic, have been specifically studied in dual-diagnosed patients (psychiatric disorder and alcohol dependence) and may confer decreased alcohol consumption and cravings. ${ }^{32}$ Quetiapine has also been studied for bipolar disorder, depression, generalized anxiety disorder, and schizophrenia. Available data are mixed, but quetiapine may be a consideration in alcohol-dependent patients with a comorbid psychiatric diagnosis, with its effect, in part, from promoting stability of the co-occurring psychiatric diagnosis. ${ }^{32-34}$

\section{Conclusion}

Currently published literature involving baclofen for the maintenance of alcohol abstinence is limited and difficult to apply to many patient populations. However, trials are currently ongoing, and the role of baclofen should become clearer as results are made known. Clinicians should be aware of both the risks and benefits associated with baclofen and its selection over other second-line agents. Selection of an agent for this indication should be based on patient characteristics, including organ function and comorbid diseases, specifically psychiatric illnesses and seizure disorders of any type.

Although the literature is not sufficient to recommend baclofen as a first-line agent for the treatment of alcohol dependence, clinicians should maintain awareness of new and novel uses of medications for alcohol dependence. Though data are inconclusive, there is some suggestion that baclofen 30-60 mg daily may be safe and effective for the treatment of alcohol-dependent individuals with normal renal function, 
with or without advanced liver disease, who have not been diagnosed with a psychiatric illness and do not carry a history of seizures.

\section{Disclosure}

No financial support was received for this publication. The authors of this paper have no sources of financial support relevant to this publication. This paper (in part or in whole) has not been previously presented or published.

\section{References}

1. World Health Organization. Global status report on alcohol and health. 2011. Geneva, Switzerland: World Health Organization; 2011.

2. Friedmann PD. Clinical practice. Alcohol use in adults. $N$ Engl J Med. 2013;368:365-373.

3. Muzyk AJ, Rivelli SK, Gagliardi JP. Defining the role of baclofen for the treatment of alcohol dependence. CNS Drugs. 2012;26(1):69-78.

4. Anton RF, O'Malley SS, Ciraulo DA, et al. Combined pharmacotherapies and behavioral interventions for alcohol dependence: the COMBINE study: a randomized controlled trial. JAMA. 2006;295(17): 2003-2017.

5. Mann K, Lehert P, Morgan MY. The efficacy of acamprosate in the maintenance of abstinence in alcohol-dependent individuals: results of a meta-analysis. Alcohol Clin Exp Res. 2004;28(1):51-63.

6. Fuller RK, Branchey L, Brightwell DR, et al. Disulfiram treatment of alcoholism. A Veterans Administration cooperative study. JAMA. 1986;256(11):1449-1455.

7. Rosner S, Hackl-Herrwerth A, Leucht S, Vecchi S, Srisurapanont M, Soyka M. Opioid antagonists for alcohol dependence. Cochrane Database Syst Rev. 2010;12:CD001867.

8. Rosner S, Hackl-Herrwerth A, Leucht S, Lehert P, Vecchi S, Soyka M. Acamprosate for alcohol dependence. Cochrane Database Syst Rev. 2010;9:CD004332.

9. Johnson BA. Naltrexone long-acting formulation in the treatment of alcohol dependence. Ther Clin Risk Manag. 2007;3(5):741-749.

10. Dario A, Tomei G. A benefit-risk assessment of baclofen in severe spinal spasticity. Drug Saf. 2004;24:799-818.

11. Addorolato G, Leggio L, Cardone S, Ferrulli A, Gasbarrini G. Role of the $\mathrm{GABA}_{\mathrm{B}}$ receptor system in alcoholism and stress: focus on clinical studies and treatment perspectives. Alcohol. 2009;43(7):559-563.

12. Addorolato G, Leggio L. Safety and efficacy of baclofen in the treatment of alcohol-dependent patients. Curr Pharm Des. 2010;16(19): 2113-2117.

13. Lioresal [package insert]. East Hanover, NJ: Novartis Pharmaceuticals Corporation, Inc; 1998.

14. El-Husseini A, Sabucedo A, Lamarche J, Courville C, Peguero A. Baclofen toxicity in patients with advanced nephropathy: proposal for new labeling. Am J Nephrol. 2011;34:491-495.

15. Clinicaltrials.gov [home page on the Internet]. Maryland, MD: National Institutes of Health [updated Aug 2012; cited November 30, 2012]. http://www.clinicaltrials.gov/. Accessed May 6, 2013.

16. Addolorato G, Leggio L, Ferrulli A, et al. Effectiveness and safety of baclofen for maintenance of alcohol abstinence in alcohol-dependent patients with liver cirrhosis: a randomized, double-blind, controlled study. Lancet. 2007;370(9603):1915-1922.

17. Leggio L, Ferrulli A, Zambon A, et al. Baclofen promotes alcohol abstinence in alcohol dependent cirrhotic patients with hepatitis $\mathrm{C}$ virus infection. Addict Behav. 2012;37:561-564.

18. Garbutt JC, Kampov-Polevoy AB, Gallop R, Kalka-Juhl L, Flannery BA. Efficacy and safety of baclofen for alcohol dependence: a randomized, double-blind, placebo-controlled trial. Alcohol Clin Exp Res. 2010;34:1-9.
19. Addolorato G, Caputo F, Capristo E, et al. Baclofen efficacy in reducing alcohol craving and intake: a preliminary double-blind randomized controlled study. Alcohol Alcohol. 2002;37:504-508.

20. Addolorato G, Leggio L, Ferrulli A, et al. Dose-response effect of baclofen in reducing daily alcohol intake in alcohol dependence: secondary analysis of a randomized, double-blind, placebo-controlled trial. Alcohol Alcohol. 2011;46:312-317.

21. Baclofen. Micromedex Healthcare Series. Available from: http://www. thomsonhc.com. Accessed May 6, 2013.

22. Dore GM, Lo K, Juckes L, Bezyan S, Latt N. Clinical experience with baclofen in the management of alcohol-dependent patients with psychiatric comorbidity: a selected case series. Alcohol Alcohol. 2011;46:714-720.

23. Rolland B, Deheul S, Danel T, Bordet R, Cottencin O. A case of de novo seizures following a probable interaction of high-dose baclofen with alcohol. Alcohol Alcohol. 2012;47:577-580.

24. Evans SM, Bisaga A. Acute interaction of baclofen in combination with alcohol in heavy social drinkers. Alcohol Clin Exp Res. 2009; 33(1):19-30.

25. Krupitsky EM, Burakov AM, Ivanov VB, et al. Baclofen administration for the treatment of affective disorders in alcoholic patients. Drug Alcohol Depend. 1993;33:157-163.

26. Agabio R, Marras P, Addolorato G, Carpiniello B, Gessa GL. Baclofen suppresses alcohol intake and craving for alcohol in a schizophrenic alcohol-dependent patient: a case report. J Clin Psychopharm. 2007;27: 319-320.

27. Hillbom M, Pieninkeroinen I, Leone M. Seizures in alcohol-dependent patients: epidemiology, pathophysiology and management. CNS Drugs. 2003;17:1013-1030.

28. Leung NY, Whyte IM, Isbister GK. Baclofen overdose: defining the spectrum of toxicity. Emerg Med Australas. 2006;18:77-82.

29. Dario A, Tomei Giustino. A benefit-risk assessment of baclofen in severe spinal spasticity. Drug Safety. 2004;27:799-818.

30. Johnson BA, Rosenthal N, Capece JA, et al. Topiramate for treating alcohol dependence: a randomized controlled trial. JAMA. 2007; 298(14):1641-1651.

31. Furieri FA, Nakamura-Palacios EM. Gabapentin reduces alcohol consumption and craving: a randomized, double-blind, placebo-controlled trial. J Clin Psychiatry. 2007;68:1691-1700.

32. Martinotti G, Andreoli S, Nicola M, Di Giannantonio M, Sarchiapone M, et al. Quetiapine decreases alcohol consumption, craving, and psychiatric symptoms in dually diagnosed alcoholics. Hum Psychopharmacol. 2008;23:417-424.

33. Litten RZ, Fertig JB, Falk DE, et al. A double-blind, placebo-controlled trial to assess the efficacy of quetiapine fumerate XR in very heavydrinking alcohol-dependent patients. Alcohol Clin Exp Res. 2012;36: 406-416.

34. Moallem N, Ray LA. Quetiapine improves response inhibition in alcohol dependent patients: a placebo-controlled pilot study. Pharmacol Biochem Behav. 2012;100:490-493.

35. Addolorato G, Caputo F, Capristo E, Colombo G, Gessa G, Gabarrini G. Ability of baclofen in reducing alcohol craving and intake: II. preliminary clinical evidence. Alcohol Clin Exp Res. 2000;24(1):67-71.

36. Ameisen O. Complete and prolonged suppression of symptoms and consequences of alcohol-dependence using high dose baclofen: a selfcase report of a physician. Alcohol Alcohol. 2004;2(40):147-150.

37. Bucknam W. Suppression of symptoms of alcohol dependence and craving using high-dose baclofen. Alcohol Alcohol. 2006;2(42):158-160.

38. Flannery B, Garbutt J, Cody M, et al. Baclofen for alcohol dependence: a preliminary open-label study. Alcoholism. 2004;10(28):1517-1523.

39. Pastor A, Jones D, Currie J. High-dose baclofen for treatment-resistant alcohol dependence. J Clin Psychopharmacol. 2012;2(32):266-268.

40. Rigal L, Alexandre-Dubroeucq C, de Beaurepaire R, Le Jeunne C, Jaury P. Abstinence and "low-risk" consumption 1 year after the initiation of high-dose baclofen: a retrospective study among "highrisk" drinkers. Alcohol Alcohol. 2012;4(47):439-442. 


\section{Publish your work in this journal}

Clinical Pharmacology: Advances and Applications is an international, peer-reviewed, open access journal publishing original research, reports,

reviews and commentaries on all areas of drug experience in humans.

The manuscript management system is completely online and includes

a very quick and fair peer-review system, which is all easy to use.

Submit your manuscript here: http://www.dovepress.com/clinical-pharmacology-advances-and-applications-journal
Visit http://www.dovepress.com/testimonials.php to read real quotes from published authors. 